\title{
Evaluation of Idiopathic Scoliosis based on Alignment, Equilibrium and Stability: Gomez Orthotic Spine System
}

\section{Jose Miguel Gomez Torres*}

Gomez Orthotic Spine Systems, St. Petersburg, Florida, USA

\begin{abstract}
Gomez Orthotic Spine Systems is a clinical method of measurement and, conservative treatment used in the management of spinal deformities including idiopathic scoliosis. This method uses easily accessible and economic measurement tools and permits a quantitative postural evaluation by using photometry for 3D analysis based on the center line in each corporal plane, line which is established as the line of greatest stability. In this article the method will be discussed in detail from its theoretical basis through implementation using a single clinical case.
\end{abstract}

\section{Terminology}

Alignment: The placement or maintenance of body structures in their proper anatomic positions, such as straightening of the teeth or repair of a fractured bone.

Equilibrium: A condition in which all influences acting upon it are canceled by others, resulting in a stable, balanced, or unchanging system.

Stability: Capacity to provide support; firmness in position.

Segments: One of the parts into which something can be divided (in this case the body).

Flexibility: The ability to readily adapt to changes in position or alignment: may be expressed as normal, limited, or excessive.

\section{Introduction}

Conservative treatment, by means of orthopedic elements for the spine has been used for many more years than surgical treatment, especially in the management of Idiopathic Scoliosis (IS). Unfortunately, there is not sufficient published scientific research that supports the conservative management of IS [1], which has given more and more room for surgical treatment. In general terms the treatment standards in orthotics for the management of IS are based in great percentage on radiographic studies including the Cobb angle [2], Risser Sign [24] and apical vertebral rotation [5,6], all of which are calculated in a bi-dimensional view unlike the three dimensional reality of scoliotic deformities giving inconclusive and partial results. Additionally, it is not of common practice for patients with IS to have full body $\mathrm{x}$-rays taken, eliminating the importance of the lower extremities in the study of alignment. This lack of information over my 26 years of professional experience has lead me to study deeply the basic principles of alignment, equilibrium and stability, and to use a three-dimensional approach to understanding their posture as the basis for individual treatment.

\section{Analysis and Solution}

I designed Gomez Orthotic Spine System (GOSS) method over 10 years ago to overcome the obstacles I faced with on a daily basis to correctly treat IS from sub-par evaluation methods. The GOSS method is based on the treatment of the patient as a whole unit or structure, viewed much in the same way as viewed by engineers and begins with the understanding of quantifying alignment ${ }^{*}$, the capacity for the equilibrium* of the patient and, most importantly, the understanding of the stability* of the body as a whole, as well as the involved segments*. Unlike traditional methods GOSS acquires all patient data through the implementation of an established protocol which requires the photo documentation of static standing posture in three planes as well as the grade of spinal flexibility followed by the determination of the corrective shape for each patient. Photometry techniques have been shown to have good inter rater and intra rater reliability [7]

We can more clearly visualize the differences between understanding a person's standing posture from a 3D approach using photometry vs. the $2 \mathrm{D} x$-ray approach by analyzing full body patient $\mathrm{x}$-rays (Figure 1). For this patient we can see three different possible reference lines, the coronal central line (CCL), represented in red, the sacral central line (SrCL) [8] represented in blue, and the C7 plumb line (C7PL) [9] represented in yellow, from which we can interpret her standing posture. The CCL is the ascending line that emerges from the central point of the base of support. Based on this line we can conclude that this patient is mal-aligned (orin a state of disequilibrium) towards the left representing approximately a $2 \mathrm{~cm}$ difference from the CCL to the SrCL, ascending line which emerges from the central point of the sacrum, and is mal-aligned $3 \mathrm{~cm}$ toward the left from the CCL to the C7PL, descending line emerging from the spinal process of C7. The SrCL and C7PL do not give exact information regarding the global alignment of the patient. In fact, according to the C7PL this patient appears to be in
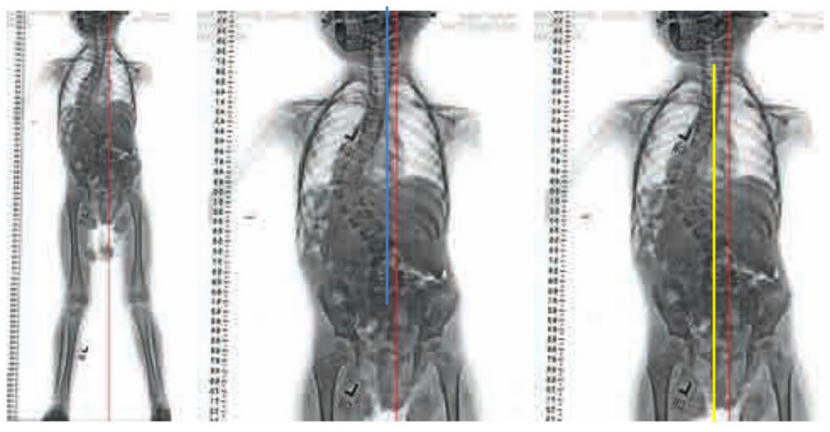

Figure 1: Reference system lines.

*Corresponding author: Jose Miguel Gomez Torres, Gomez Orthotic Spine Systems, St. Petersburg, FL, USA, Tel: 713-8700662; E-mail: jmgomezmd@gorthoticsystems.com

Received October 24, 2013; Accepted December 10, 2013; Published December 12, 2013

Citation: Torres JMG (2013) Evaluation of Idiopathic Scoliosis based on Alignment, Equilibrium and Stability: Gomez Orthotic Spine System. J Spine 2: 149. doi:10.4172/2165-7939.1000149

Copyright: (c) 2013 Torres JMG. This is an open-access article distributed under the terms of the Creative Commons Attribution License, which permits unrestricted use, distribution, and reproduction in any medium, provided the original author and source are credited. 
adequate alignment and therefore a treatment implemented based on this interpretation would focus only on reducing the curve magnitude and would not consider the overall alignment of the patient, treatment based on the postural interpretation according to the CCL. In the GOSS protocol, an overall mal-alignment of $3 \mathrm{~cm}$ in the case of idiopathic scoliosis is considered a severe deformity of potential greater risk than the scoliotic curve presented by the patient in the $\mathrm{x}$-ray.

At this point, I would like to share the steps used in the GOSS protocol for the adequate understanding of three-dimensional deformities that are present in different body segments, which affect to a greater degree patients with spinal deformities. In the evaluation of each patient using GOSS principles, photos are taken of the coronal plane in anterior and posterior views (Figure 2), the sagittal plane in right and left views (Figure 2), and the transverse plane. Two types of photos are taken of the transverse plane: 1 . In the Adams test $[10,11]$ position in proximal and distal views (Figures 2 and 3). A photo in standing taken from the superior view from the head looking downward to evaluate the rotation in the involved segments (Figure 4). With these photos, we focus on understanding the clinical signs and quantifying the deformity. All these photos should be taken with a grid in the background and a floor mat (in the form of a " $\mathrm{T}$ ") used for the equidistant positioning of the feet, also, positioning adequately the tripod and laser in order to locate the lines of maximal stability in each plane, Coronal Center Line (CCL), Sagittal Center Line (SCL) and Transverse Center Line (TCL). The next step is to create the corrective position which depends on the characteristics of the scoliotic curve/s through documentation using

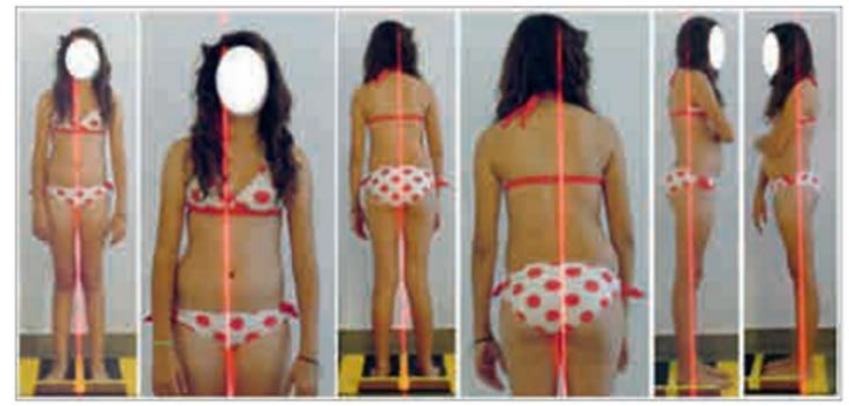

Figure 2: Coronal and sagital plane photometry.

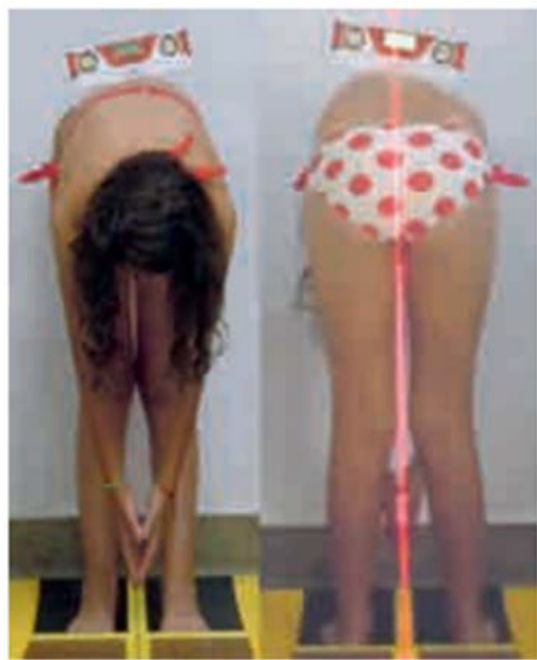

Figure 3: Transverse plane photometry: Adams Test.

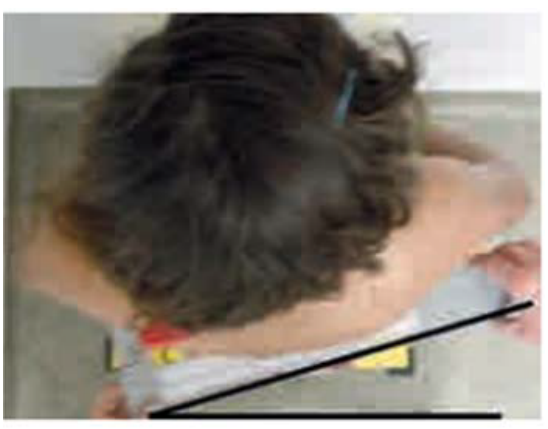

Figure 4: Transverse plane photometry.

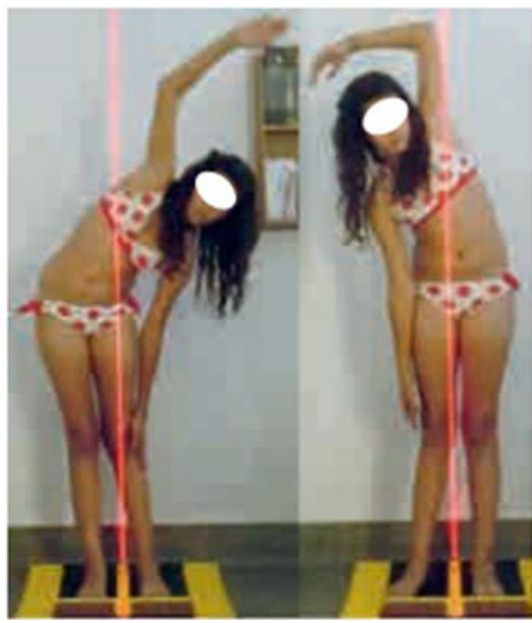

Figure 5: Lateral flexibility.

various photos and videos (as necessary) which suggest the degree of flexibility [5] of the curve/s. Following the photo documentation from each case we analyze the photos to determine the alignment of each plane. I will use the analysis of the case of a 12 year old female patient with the diagnosis of Adolescent Idiopathic Scoliosis (AIS) to demonstrate this process (Figures 2-7).

\section{Coronal plane-anterior view (Figure 2)}

It is having adequately obtained the CCL we can conclude that the patient is in left mal-alignment of approximately $3 \mathrm{~cm}$. The lumbarpelvic segment has an inclination in the clockwise direction, which brings us consider a leg length discrepancy, being the left leg which is shorter, or the presence of a pelvic inclination coming from the curve.

\section{Coronal plane-posterior view (Figure 2)}

We visualize the CCL using the laser and confirm that the patient has disequilibrium towards the left.

\section{Sagittal plane-right side view (Figure 2)}

The SCL is determined beginning at the anterior border of the lateral malleolus. In this case it is evident that the patient is mal-aligned anteriorly approximately $5 \mathrm{~cm}$. The lumbar-pelvic segment has an anterior inclination which, along with the hyperextension in the knees, is the cause of the sagittal mal-alignment.

\section{Sagittal plane-left side view (Figure 2)}

The SCL passes through the left shoulder (unlike in the photo of the 


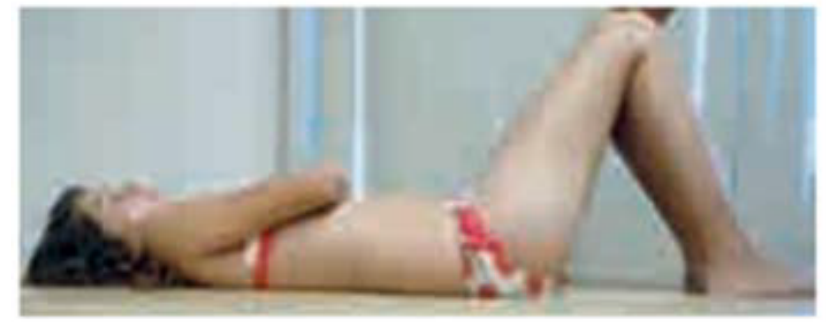

Figure 6: Coronal plane corrective shape.

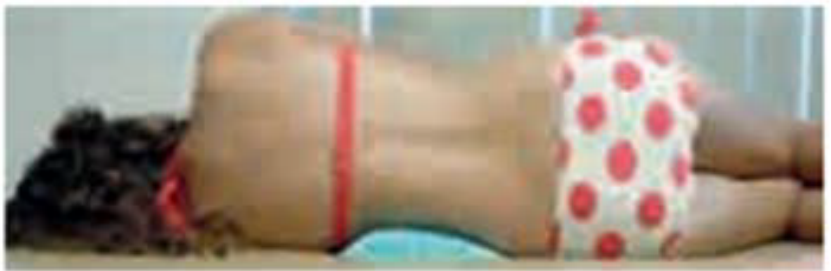

Figure 7: Sagital plane corrective shape.

right side), which is an indication of a rotation in the counter-clockwise direction at the level of the shoulders.

\section{Transverse plane (Adams Test [10,11]) (Figure 3)}

This test should be done with the patient in standing, asking them to flex the trunk forward over the pelvis (without bending the knees). In the initial phase, we look for thoracic rotational deformities and the closer the patient becomes to touching their toes we can see the presence of thoracic-lumbar and lumbar deformities. A scoliometer or level should be used to measure the maximal segmental rotation (usually at the apex of the curves).

\section{Transverse plane (Adams Test $[10,11]$ ) - proximal view) (Figure 3)}

$8^{\circ}$ of thoracic-lumbar segment rotation in the counter-clockwise direction

\section{Transverse plane (Adams Test $[10,11])$ - distal view) (Figure 3)}

$13^{\circ}$ of thoraco-lumbar segment rotation in the clockwise direction

Note: This information can be confused by professionals because the reality is that the transverse plane should always be evaluated from the head downward (proximal view). In spite of the fact that the level is positioned at very similar point, in both photos, we obtain results with a difference of $5^{\circ}$ (which is not exact). Unfortunately, in my opinion, the Adams test is not precise; it changes the sagittal plane by elongating it lessening the degrees of rotation in the transverse plane when the rotational component is less than approximately $20-25^{\circ}$ or it increases the degrees of rotation when the rotational component is greater than $25^{\circ}$. For this reason I recommend evaluating the rotations in the following manner (from proximal to distal).

\section{Transverse plane (proximal to distal) (Figure 4)}

In a neutral standing position, an anthropometric calibrator, that can be blocked, is used to view in a more precise way the transverses plane characteristics of the different segments. In this case, at the same thoraco-lumbar level in which the scoliometer was placed we obtain $17^{\circ}, 4^{\circ}$ more rotation in comparison with the Adams test. In reality, this deformity is more aggressive and structured than that established by the previous analysis. The transverse plane is the plane which structures the curves, is responsible for the loss of flexibility as well as the possibility of correction of the spinal deformities.

With this information I invite you to resume the presentation in the 3 planes: Coronal- $3 \mathrm{~cm}$ left mal-alignment. Sagittal- $5 \mathrm{~cm}$ anterior malalignment. Transverse- $17^{\circ}$ counter-clockwise rotation in the thoracolumbar segment. In your opinion, which plane is the most involved?

Yes! You are right. The transverse plane is the most involved, the sagittal plane is the second most involved for its anterior disequilibrium and the coronal plane is the least involved for its left disequilibrium. So, why do we always treat the coronal plane as if it were the most involved and focus so much on the Cobb angle. This evaluation method does not give us the correct information about the deformity.

If you are following the details of the GOSS system you already have the numerical information regarding the mal-alignments $(\mathrm{cm})$ and rotations (degrees) of the deformity. Now, with the same patient, we need to analyze and measure the corrective shape of the deformity. This is done using the factor of flexibility [12] and correction without the need to manipulate the patient with force to unbend the curves. We should ask the patient, from a standing position, to perform a maximal lateral flexion to the left and to the right in order to understand which side has the greatest range of movement and flexibility in the coronal plane (Figure 5). In this case, there is no doubt that it is the left side. Equally, the patient does a maximal forward flexion and extension to evaluate the sagittal plane. Also, maximal rotation in a clockwise and counter-clockwise direction is done to evaluate the transverse plane in standing and sitting.

The second step is to place the patient on a plinth to create the most aggressive corrective shape possible using the force of gravity where we can determine quantitatively the flexibility of the deformity (Figures 6 and 7). In order to meet this objective, the patient needs to be in side lying on the side of the convexity of the curve (in this case the left side). We begin with the correction of the sagittal plane (Figure 6) by flexing the hip and knee joints to $45^{\circ}$, which permits a posterior pelvic inclination. Likewise, we make the correction by anchoring the pelvis and the trunk below the axilla. The second plane we treat is the transverse plane performing a de-rotation in the direction contrary to the direction of the deformity in the most involved segment. To treat the $17^{\circ}$ thoraco-lumbar rotation in the counter-clockwise direction, a de-rotation force must be applied in a clock-wise direction up to or less than $17^{\circ}$.

The last plane we treat is the coronal plane in which the corrective forces of the other 2 planes are sustained or conserved and a lateral bending moment is applied by the use of a half-bolster (Figure 8) that is positioned to exercise maximum pressure at the apex of the curve

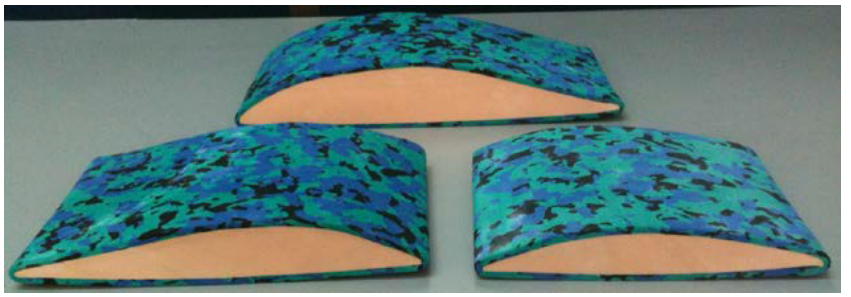

Figure 8: Half- Bolsters used to provide lateral bending moment. 


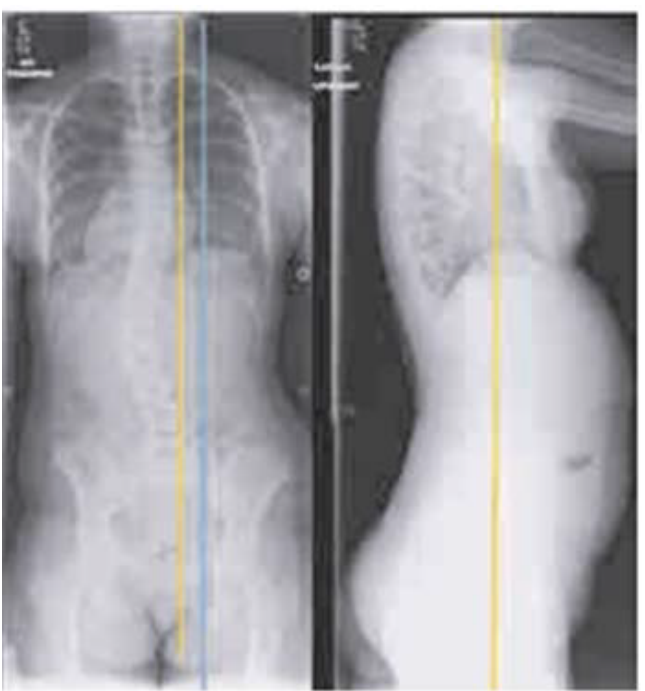

Figure 9: Radiological Documentation.

(L1-L2) (Figure 7). For more than three points of pressure we should use a lateral bending moment. Equally, the position in supine in hip and knee flexion provides valuable information in understanding the flexibility of the sagittal and transverse planes. We should not forget that the sagittal plane has physiological curves that need to be maintained or created during the correction. In both of the mentioned positions measurements should be taken (circumference, medio-lateral, anteriorposterior and length) while maintaining the patient in the position of corrective shape, not the position of deformity.

Following this exercise, it is time to analyze the radiographic information (Figure 9) and compare it to our clinical and mechanical information. As you can see, the sagittal alignment, using the $\mathrm{x}$-rays, is normal. However, we remember that the patient presents an anterior mal-alignment of $5 \mathrm{~cm}$, information which the sagittal X-ray does not correctly provide. We do not have a CCL or a SCL and as always we must work with the C7PL (yellow) or the SrCL (blue). As you can see, the clinical signs based on the photos and the $\mathrm{x}$-rays are not in accordance. For this reason, we must concentrate on evaluating the patient as a whole and not by $\mathrm{x}$-rays only.

The information acquired during the GOSS method evaluation is then used in conjunction with CAD/CAM technology to design an asymmetrical brace for an individualized conservative treatment of IS based on the patient's true alignment, equilibrium and overall body stability.

\section{Conclusion}

The evaluation of IS using bi-dimensional techniques and providing treatment based on the same lacking techniques continues to be an existing problem in the field of orthotics today. Evaluation of IS from a 3D full-body approach can easily be performed in a clinical setting and provides more comprehensive understanding of the patients spinal deformity based on their ideal overall posture and balance. This form of evaluation, established in the GOSS method, provides a strong foundation for IS treatment with conservative bracing as the evaluation results can be transformed into the design of a 3D bracing system.

\section{References}

1. Stokes OM, Luk KD (2013) The current status of bracing for patients with adolescent idiopathic scoliosis. Bone Joint J 95-95B: 1308-16.

2. Negrini S, Aulisa AG, Aulisa L, Circo AB, de Mauroy JC et al. (2012) 2011 SOSORT guidelines: Orthopaedic and Rehabilitation treatment of idiopathic scoliosis during growth. Scoliosis $7: 3$.

3. Hacquebord JH, Leopold SS (2012) In brief: The Risser classification: a classic tool for the clinician treating adolescent idiopathic scoliosis. Clin Orthop Relat Res 470: 2335-2338.

4. Nault ML, Parent S, Phan P, Roy-Beaudry M, Labelle H, et al. (2010) A modified Risser grading system predicts the curve acceleration phase of female adolescent idiopathic scoliosis. J Bone Joint Surg Am 92: 1073-1081.

5. Ovadia D, Eylon S, Mashiah A, Wientroub S, Lebel ED (2012) Factors associated with the success of the Rigo System Chêneau brace in treating mild to moderate adolescent idiopathic scoliosis. J Child Orthop 6: 327-331.

6. Lebel DE, Al-Aubaidi Z, Shin EJ, Howard A, Zeller R (2013) Three dimensional analysis of brace biomechanical efficacy for patients with AIS. Eur Spine J 22 . 2445-2448.

7. Fortin C, Feldman DE, Cheriet F, Labelle H (2011) Clinical methods for quantifying body segment posture: a literature review. Disabil Rehabil 33: 367383.

8. Guo J, Liu Z, Qian B, Zhu Z, Jiang H, et al. (2012) Anatomic deviation between the central hip vertical axis and central sacral vertical line in adolescent idiopathic scoliosis. J Spinal Disord Tech 25: E161-166.

9. Grosso C, Negrini S, Boniolo A, Negrini AA (2002) The validity of clinical examination in adolescent spinal deformities. Stud Health Technol Inform 91: 123-125.

10. Fairbank J (2004) Historical perspective: William Adams, the forward bending test, and the spine of Gideon Algernon Mantell. Spine (Phila Pa 1976) 29: 19531955.

11. Hackenberg L, Hierholzer E, Bullmann V, Liljenqvist U, Götze C (2006) Rasterstereographic analysis of axial back surface rotation in standing versus forward bending posture in idiopathic scoliosis. Eur Spine J 15: 1144-1149.

12. Chen ZQ, Zhao YF, Bai YS, Liu KP, He SS, et al. (2011) Factors affecting curve flexibility in skeletally immature and mature idiopathic scoliosis. J Orthop Sci 16: $133-138$. 